\title{
VLF and HF Plasma Waves Associated with Spread-F Plasma Depletions Observed on the C/NOFS Satellite
}

\author{
R. Pfaff, H. Freudenreich, P. Schuck, J. Klenzing
}

\author{
NASA/Goddard Space Flight Center, Greenbelt, MD, USA, (Robert.F.Pfaff@nasa.gov)
}

The C/NOFS spacecraft frequently encounters structured plasma depletions associated with equatorial spread-F along its trajectory that varies between $401 \mathrm{~km}$ perigee and $867 \mathrm{~km}$ apogee in the low latitude ionosphere. We report two classes of plasma waves detected with the Vector Electric Field Investigation (VEFI) that appear when the plasma frequency is less than the electron gyro frequency, as is common in spread-F depletions where the plasma number density typically decreases below $10^{4} / \mathrm{cm}^{3}$. In these conditions, both broadband VLF waves with a clear cutoff at the lower hybrid frequency and broadband HF waves with a clear cutoff at the plasma frequency are observed. We interpret these waves as "hiss-type" emissions possibly associated with the flow of suprathermal electrons within the inter-hemispherical magnetic flux tubes. We also report evidence of enhanced wave "transients" sometimes embedded in the broader band emissions that are associated with lightning sferics detected within the depleted plasma regions that appear in both the VLF and HF data. Theoretical implications of these observations are discussed. 